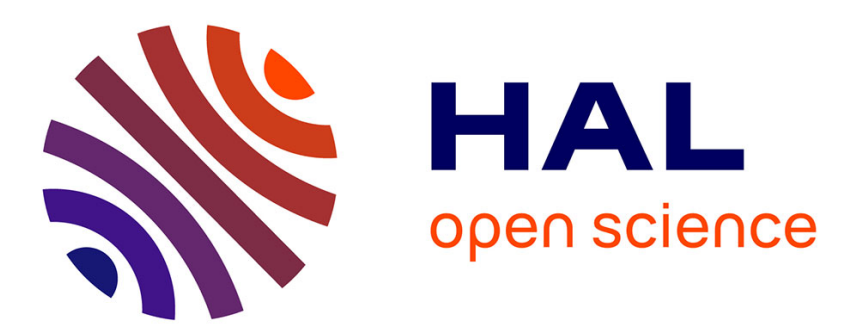

\title{
Dynamic adaptation of rules bases under cognitive constraints
}

\author{
Philippe Lenca, Elisabeth Le Saux, Philippe Picouet
}

\section{To cite this version:}

Philippe Lenca, Elisabeth Le Saux, Philippe Picouet. Dynamic adaptation of rules bases under cognitive constraints. Proceedings of the conference human centered processes (HCP'99), Sep 1999, Brest, France. pp.277-291. hal-02161207

\section{HAL Id: hal-02161207 https://hal.science/hal-02161207}

Submitted on 27 Jul 2021

HAL is a multi-disciplinary open access archive for the deposit and dissemination of scientific research documents, whether they are published or not. The documents may come from teaching and research institutions in France or abroad, or from public or private research centers.
L'archive ouverte pluridisciplinaire HAL, est destinée au dépôt et à la diffusion de documents scientifiques de niveau recherche, publiés ou non, émanant des établissements d'enseignement et de recherche français ou étrangers, des laboratoires publics ou privés. 
DYNAMIC ADAPTATION OF RULES BASES UNDER COGNITIVE CONSTRAINTS

\author{
Philippe Lenca, Elisabeth Le Saux, Philippe Picouet ${ }^{1}$ \\ ${ }^{1}$ Département IASC - ENST Bretagne - BP 832 \\ F-29285 BREST CEDEX
}

\begin{abstract}
:
In the framework of the COMAPS (COgnitive Management of Anthropocentric Production Systems) project (Brite Euram BE 96-3941) this paper presents an algorithm dedicated to the updating of a rules base under cognitive constraints. These constraints come from the assumption that the rules are reasonable "approximations" of those used by a human expert.
\end{abstract}

Keywords: cognitive modeling, knowledge acquisition, process control

\title{
1 Introduction
}

This paper is dedicated to a cognitive approach for industrial process control and to its use for designing an expert decision support system: the COMAPS tool. The COMAPS tool is based on a rather simple observation about the life cycle of man-machine cooperation in the management of an industrial process [Barthélemy et al., 1995]. Such a cycle can be decomposed into three periods (fig. 1). During the learning period, the human operator comes from the state of novice to the state of expert [Shanteau, 1988]. In the maintenance phase, the expert operator applies his/her know-how and adapts his/her rules to control the process. At the revision period (breaking period), either the expert and the physical system have evolved in drastically different ways or some important structural changes occur. In any case, simple adaptation of rules is not enough any more and a learning phase has to be initialized once more.

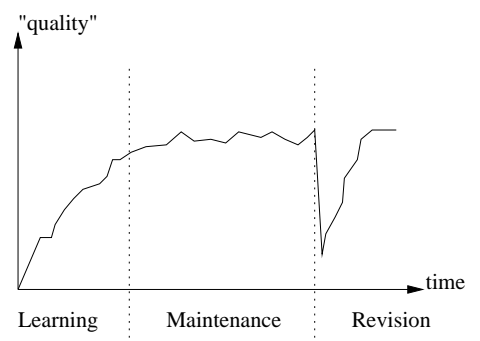

Figure 1: Life cycle of a process

Such a scheme inscribes in the framework of Anthropocentric Production Systems [Wobbe, ] i.e. forms of advanced manufacturing which depend upon a balanced 
integration between human skills, collaborative work organisation and adapted technologies. This kind of approach is also a way to capitalize knowledge and to adapt transfers of expertise.

The COMAPS tool is itself articulated into three phases: an offline learning phase, an online maintenance phase and a conflict solving phase. The learning phase extracts a set of initial rules from a sample. The learning algorithm is based on decision tree paradigm [Müller and Wiederhold, 1999]. The maintenance phase updates the rules according to new incoming information [Le Saux et al., 1999]. The conflict solving phase [Saunier and Bisdorff, 1999] is called when no acceptable modification can be recommended by the maintenance phase algorithm. This part of the COMAPS tool is the one that needs to fully interact with the expert operator and all the COMAPS manmachine interface is designed conjointly with it.

This paper emphasizes on the second phase. In section 2, we first describe the cognitive model we shall use; in section 3 , we discuss some formalization issues. The main features of the maintenance phase are presented in section 4 and some concrete results are discussed in section 5 .

\section{Cognitive and methodological approach}

\subsection{Methodological aspects}

Our methodology is based on an on-line non intrusive acquisition of the operator's behavioural strategies. It involves three main features:

1. The expertise modelling and the strategies extraction techniques follow cognitive principles like bounded rationality [Simon, 1979], parcimony [Barthélemy and Mullet, 1986] ...

2. The expert operator is in the loop of the process control, even in the aim of the strategies convergence towards a meaningful set of rules.

3. The protocol and the algorithm techniques are specific to the incremental and iterative aspects underlying the maintenance phase.

In the COMAPS framework, the expert is directly observed, on real situations, when performing decision making tasks.

\subsection{Cognitive model}

In order to learn the decision maker's strategies, we follow Montgomery's principle of search for a dominance structure [Montgomery, 1983] instancied as the moving basis heuristics (MBH) [Barthélemy and Mullet, 1986, Barthélemy and Mullet, 1992].

The cognitive model assumes that the decision maker (DM) shows rationality in the way that something is optimized. But this rationality is bounded by his/her cognitive abilities (stocking and computing in a short term working memory) and his/her satisfaction features. As a consequence, he/she uses a not too large collection of stable strategies but involving a small amount of information. These strategies are assumed to be stored in a long term memory. They have been constructed from the DM experience. 
In addition, the DM is supposed to use some combinations of information rather more frequently than others.

According to the above observations, the MBH integrates three main cognitive principles:

- parsimony:

due to his/her short-term memory capacity, the DM manipulates a small amount of information [Aschenbrenner and Kasubek, 1978, Johnson and Payne, 1985],

- reliability/warrantability: the processed information that leads to a choice has to be large enough (in quantity and/or quality) for individual and/or social justification [Montgomery, 1983, Ranyard and Crozier, 1983],

- decidability/flexibility: the DM decides after a sequence of changes in term of processed information until a decision is made at a relatively short notice [Huber, 1986, Montgomery, 1983, Svenson, 1979].

\subsection{Algorithmic consequences}

As a consequence of the MBH, COMAPS tool searchs for classical rules: "if condition then decision". Main differences with the usual machine learning approach are that the "condition" involves always a small amount of information and that the expert does not use a "large" set of rules.

\section{Formalisation}

\subsection{Data and rules}

We are concerned by:

- A set $X=\left\{X_{1}, \ldots, X_{p}\right\}$ of $p$ control parameters. Each parameter $X_{i}$ has a values domain (numerical or nominal) $V_{i}$. The global domain is the set $V=\prod_{1 \leq i \leq p} V_{i}$.

- A set $Q=\{1, \ldots, q\}$ of labels denoting the decision outcomes. An element $x$ of $V \times Q$ is called a control situation $(c s)$.

- A rule is tuple $R=\left(W_{i_{1}}, \ldots, W_{i_{q}} ; j\right)$ with $W_{i_{k}} \subseteq V_{i_{k}}$, and $j \in Q$. The rule $R$ will be sometimes written under a conjunctive form as: $\left[\left(X_{i_{1}} \in W_{i_{1}}\right) \wedge \ldots \wedge\left(X_{i_{q}} \in\right.\right.$ $\left.\left.W_{i_{q}}\right)\right] \rightarrow j$. The first term is the condition of $R$ and the second term its label. The dimension, $\operatorname{dim}(R)$, of a rule $R$ is the number of control parameters occuring in its condition.

Let $H \subseteq V \times Q$ be a set of $c s$ and $B$ be a set of rules we say that:

- The $\operatorname{cs} x=\left(x_{1}, \ldots, x_{p} ; l\right)$ is covered by the rule $R=\left(W_{i_{1}}, \ldots, W_{i_{q}} ; j\right)$ whenever $x_{i_{k}} \in W_{i_{k}}$. If moreover, $l=j, x$ is said to be well covered by $R$.

- $B$ applies on $H$ whenever each $R \in B$ covers at least one $c s$ in $H$ and each $x \in H$ is covered by at least one rule in $B$. $B$ is consistent with $H$ whenever $B$ applies on $H$ and every $x \in H$ is well covered by some rule in $B$. 
We can restate the COMAPS problematic in term of updating a set of rules $B$ and a set of $c s H$ under three constraints: $\left(C_{1}\right)$ : each rule has a "small" dimension; $\left(C_{2}\right)$ : the number of rules is "not too large"; $\left(C_{3}\right)$ : the rules constitute a set which is consistent with the current set of $c s$.

\subsection{Complexity issues}

The conditions $C_{1}, C_{2}, C_{3}$ above correspond to NP-complete problems (for NPcompleteness issues we follow the terminology from [Garey and Johnson, 1979]).

Theorem - Let $v \geq 2$ be an integer. The following problem is NP-complete:

Name[v-COMAPS] (v-dimensional-COMAPS)

Instance: a set $H$ of $c s$, an integer $m$.

Question: does it exist a set $B$ of rules such that $B$ is consistent with $H,|B| \leq m$ and $\operatorname{Max}_{R \in B} \operatorname{dim}(R) \leq v ?$

Sketch of proof: the set of $c s$ covered by the rule $R=\left(x_{i_{1}} \in W_{i_{1}} \wedge \ldots \wedge x_{i_{k}} \in W_{i_{k}} \rightarrow j\right.$ constitutes a so-called cylinder of $H$ with basis $W_{i_{1}} \times \ldots \times W_{i_{k}}$. The set of rules $B$ is consistent with $H$ if and only if the corresponding cylinders constitute a partition of $H$. The result is then obtained, with standard arguments, by reduction from the clique partitioning problem in graphs [Garey and Johnson, 1979]).

This remark assigns to the maintenance phase another role: correct eventual errors from the learning phase and correct its own errors ...

\subsection{Some relaxations}

In fact conditions $C_{1}, C_{2}$ and $C_{3}$ appear as much too strong. In particular:

1. some inconsistencies (i.e. $c s$ covered by a rule $R$, but not well-covered by $R$ ) can appear sporadically without seriously affecting the robustness of a rules set.

2. "waiting before updating", in order to find an efficient solution, could be better strategy than "change the rule at the first observed inconsistency".

3. some observed inconsistencies can be just the result of priority between rules. This is the case when the CS $x$ is well-covered by $R$, covered by $R^{\prime}$ and the DM uses $R$ prioritary to $R^{\prime}$.

To account for these three remarks, we introduce a priority relation $\Pi$ on a rules set applying to $H$; the notion of outer-covered $c s$, a certainty factor $\gamma(R)$ of the rule $R$.

- $\Pi$ is a reflexive, acyclic relation on $B$

- $x \in H$ is outer covered by $R \in B$ whenever $x$ is covered by $R$ and if $x$ is wellcovered by $R^{\prime}$ then $R^{\prime} \Pi R\left(R^{\prime}\right.$ has priority on $R$ ).

- $\gamma(R)=\frac{\mid\{x \in H: x \text { is well covered by } R\} \mid}{\mid\{x \in H: x \text { is covered by } R\} \mid}$

Another notion, Promising Combination of Aspects, is also usefull to increase the efficiency of the maintenance phase. It is discussed in [Lépy, 1999]. In any case, with these materials, condition $C_{3}$ can be weakened into: $\left(C_{3}^{\prime}\right) B$ applies on $H$ and $\operatorname{Max}_{R \in B} \gamma(R) \leq \sigma$ with $\sigma \leq 1$ a threshold.

HCP'99

Brest, France, September 1999 


\section{Maintenance algorithm}

We shall describe this algorithm in a rather approximate way (but sufficient to understand its main features). For more substantial details the reader can refer to [Le Saux et al., 1999].

\subsection{Principles}

The algorithm starts with a set $H_{0}$ of $c s$, a set $B_{0}$ of rules and a priority relation $\Pi_{0}$ on $B_{0}$ fulfilling the conditions $C_{1}, C_{2}$ and $C_{3}^{\prime}$. According to new incoming $c s$ arriving at time $1, \ldots, t, t+1, \ldots$ that added to $H_{0}$ constitute the sequence $H_{0}, H_{1}, \ldots, H_{t}, \ldots$ of histories, it updates the rules and the priority relation in order to get a sequence $B_{0}, B_{1}, \ldots, B_{t}, \ldots$; $\Pi_{0}, \Pi_{1}, \ldots, \Pi_{t}, \ldots$ such that any triple $\left(H_{t}, B_{t}, \Pi_{t}\right)$ satisfies to $C_{1}, C_{2}$ and $C_{3}^{\prime}$ (algorithmic updating of $H_{t}$ and some other points are omitted).

\subsection{Updating the rules base}

Three cases can occur when a new $\operatorname{cs} x$ arrives:

1. $x$ is well-covered by at least one rule $R$ and badly covered by none.

2. no rule in $B_{t}$ covers $x$,

3. $x$ is badly covered by at least one rule $R$ in $B_{t}$.

In the first case, set $H_{t+1}=H_{t} \cup\{x\}, B_{t+1}=B_{t}, \Pi_{t+1}=\Pi_{t}$.

In the cases 2 and 3, the algorithm uses, hierarchically, six functions that have been implemented to adapt $B_{t}$ :

1. generalize an existing rule to cover a set of CS that are not or badly covered, by the following operations: removal of one dimension over a rule to extend it, extension the domain of a control parameter occuring in the rule.

2. create a new rule to cover a set of $c s$ that are not or badly covered.

3. from a badly covering rule, create a "subrule" on the same control parameters as the initial rule ones in order to cover the $c s$ well.

4. create a new rule with control parameters complementary to parameters involved in the bad covering rules.

5. add a new control parameter and its values domain in a rule $R$.

6. change the priorities between the rules.

The parameters of the algorithm are the maximum dimension $m$ of a rule and the threshold $\sigma$. The operations (1) up to (6) are tested hierarchically, the first one for which $\operatorname{Max} \gamma(R) \leq \sigma$ and $\operatorname{Max} \operatorname{dim}(R) \leq m$ is used to update $B_{t}$. When they all fail, two possibilities remain: parameters $\sigma$ or $m$ are tuned or the third phase starts. 


\section{Current results}

Real data being confidential, we've been testing the algorithm through a mockup with coded data. The first tests have been led without the expert, dividing the coded data set into a training set for the learning phase and an incoming situations set for the maintenance phase.

With one of the pilot sites data, we had the possibility to test the behaviour of the maintenance phase facing a known evolution of the process control. Starting from a set of 6 institutional rules and an history of $1086 \mathrm{cs}$, the rules set has been updated according to 556 new $c s$.

The results according to the quality of the rules are summarized in Figure 2, the last column corresponding to the type of modification applied according to the list presented in 4.2. All the results have been shown to the expert and they were validated.

\begin{tabular}{|c|c|c|c|}
\hline Rule & $\gamma$ Before & $\gamma$ After & Modification \\
\hline 1 & 0.41 & 1 & \\
2 & 0.84 & 0.87 & \\
3 & 0.56 & 0.87 & 5. \\
4 & 0.57 & 0.69 & 6. \\
5 & 0.89 & 0.87 & 5. \\
6 & 0.70 & 0.88 & 5. and 6. \\
7 & & 0.84 & 4. \\
8 & & 0.86 & 4. \\
\hline
\end{tabular}

Figure 2: Quality of the rules

We wait for a prototype, now under development, to be installed for benchmarking to be able to make some more tests, but this time in a real decision context and not only with a posteriori validation.

Some comparisons between classical machine learning tools, and especially decision tree learning tools like ID3 [Quinlan, 1986] and C4.5 [Quinlan, 1993] are also under way.

\section{References}

[Aschenbrenner and Kasubek, 1978] Aschenbrenner, K. and Kasubek, W. (1978). Challenging the custing syndrome: multi-attribute evaluation of cortison drugs. Organizational Behaviour and Human Performances, (22):216-234.

[Barthélemy et al., 1995] Barthélemy, J.-P., Coppin, G., and Guillet, F. (1995). Smelting process control: from experimental design to acquisition of expertise. In International Conference on Industrial Engineering and Production Management, volume 2, pages $2-11$.

[Barthélemy and Mullet, 1986] Barthélemy, J.-P. and Mullet, E. (1986). Choice basis: a model for multi-attribute preference. British Journal of Mathematical and Statistical Psychology, (39):106-124. 
[Barthélemy and Mullet, 1992] Barthélemy, J.-P. and Mullet, E. (1992). A model of selection by aspect. Acta Psychologica, (79):1-19.

[Garey and Johnson, 1979] Garey, M. and Johnson, D. (1979). Computers and intractability, a guide to the theory of NP-completeness. New York:Freeman.

[Huber, 1986] Huber, O. (1986). Decision making as a problem solving process. In Brehmer, B., Jungermann, H., Lourens, P., and Sevon, G., editors, New directions in research on decision making.

[Johnson and Payne, 1985] Johnson, E. and Payne, J. (1985). Effort and accuracy in choice. Management Science, (31):295-314.

[Le Saux et al., 1999] Le Saux, E., Lenca, P., Picouet, P., and Barthélemy, J. (1999). An anthropocentric tool for decision suppert. In The Sixteenth International Joint Conference on Artificial Intelligence, pages 338-343, Stockholm, Sweden.

[Lépy, 1999] Lépy, N. (1999). Conditional learning. In Human Centered Processes.

[Montgomery, 1983] Montgomery, H. (1983). Decision rules and the search for a dominance structure: toward a process model of decision making. In Humphrey, P., Svenson, O., and Vari, A., editors, Analyzing and Aiding Decision Process, pages 471483, Amsterdam:North Holland.

[Müller and Wiederhold, 1999] Müller, W. and Wiederhold, E. (1999). Machine learning methodology and cognitive modelling of human expert operators in anthropocentric processes. In Human Centered Processes.

[Quinlan, 1986] Quinlan, J. R. (1986). Induction of decision trees. Machine Learning, 1(1):81-106.

[Quinlan, 1993] Quinlan, J. R. (1993). C4.5: Programs for Machine Learning. Morgan Kaufmann, San Mateo, CA.

[Ranyard and Crozier, 1983] Ranyard, R. and Crozier, R. (1983). Reasons given for risky judgment and choice: a comparison of three tasks. In Humphrey, P., Svenson, O., and Vari, A., editors, Analyzing and Aiding Decision Process, North Holland.

[Saunier and Bisdorff, 1999] Saunier, P. and Bisdorff, R. (1999). Man machine interface for a decision checking tool. In Human Centered Processes.

[Shanteau, 1988] Shanteau, J. (1988). Psychological characteristics of expert decision maker. Acta Psychologica, (91):203-215.

[Simon, 1979] Simon, H. (1979). Models of thought. Yale University Press, New Haven.

[Svenson, 1979] Svenson, O. (1979). Process description of decision making. Organizational behaviour and human performance, (23):86-112.

[Wobbe, ] Wobbe, W. Anthropocentric production systems: A strategic issue for europe. volume 1 of APS Research Paper Series (FAST). European Communities, Brussels. 